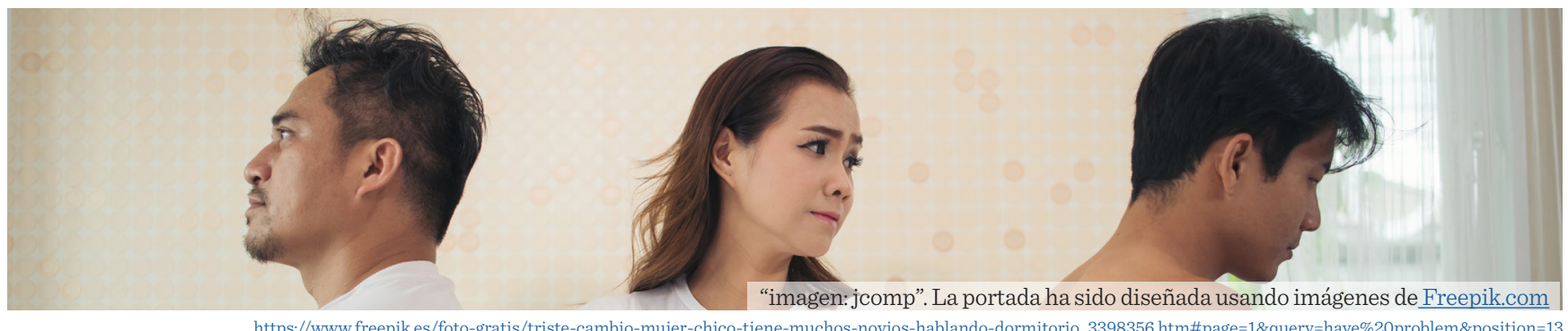

\title{
Modelo predictivo de conducta de infidelidad sexual entre personas casadas
}

\section{Predictive model of sexual infidelity behavior among married persons}

José Moral-de-la-Rubia

\section{RESUMEN}

La infidelidad marital es relativamente frecuente a pesar de sus consecuencias. Este estudio tuvo como objetivo desarrollar un modelo predictivo de la conducta sexual infiel en personas casadas, mediante el análisis de siete variables que participan en el fenómeno de la infidelidad. Se colectó una muestra de 790 personas casadas (53.4 \% mujeres y $47.6 \%$ hombres) por rutas al azar en Monterrey, México. Se aplicó la Escala de Valoración de la Relación, Indice de Satisfacción Sexual, Escala de Actitud hacia la Exclusividad Marital, Escalas de Conducta Infiel, Escala de Búsqueda de Sensaciones Sexuales y Escala de Engrandecimiento Marital. Se especificó el modelo con 2 variables exógenas correlacionadas (búsqueda de sensaciones sexuales y engrandecimiento marital) y 5 variables endógenas (actitud hacia la infidelidad, satisfacción marital, satisfacción sexual, deseo de infidelidad sexual y conducta de infidelidad sexual). De cada 100 participantes, 6 estaban insatisfechos con su matrimonio, 13 con las relaciones sexuales con su pareja y 20 veían más defectos que virtudes en sus parejas. Además, 83 informaron no incurrir en conductas de infidelidad sexual, 60 indicaron total rechazo hacia la propia infidelidad, 72 no mostraron deseo de infidelidad sexual y 51 reportaron no buscar de sensaciones sexuales. El modelo mostró un efecto grande sobre la conducta de infidelidad sexual, explicando $70.8 \%$ de su varianza, y tuvo un buen ajuste: $\chi^{2}(7)=11.93, P=0.10$, RMSEA $=0.03$ y SRMR $=0.01$. Se concluye que la variable más crítica es el deseo de infidelidad sexual, cuyo determinante más importante es la búsqueda de sensaciones sexuales. La correlación negativa entre esta última variable y engrandecimiento marital indica que estas 2 variables se pueden modular una a la otra. A su vez, el modelo remarca aspectos de insatisfacción marital y sexual.

PALABRAS CLAVE: parejas casadas, conducta de infidelidad sexual, deseo de infidelidad sexual, búsqueda de sensaciones sexuales, análisis de sendero.

\section{ABSTRACT}

Marital infidelity is relatively frequent despite its consequences. The aim of the study was to develop a predictive model of sexual infidelity behavior among married persons, through the analysis of seven variables related to the infidelity construct. A sample of 790 married persons (53.4\% women and $47.6 \%$ men) was collected in Monterrey, Mexico using random route sampling. The Relationship Assessment Scale, Sexual Satisfaction Index, Attitude toward Marital Exclusivity Scale, Unfaithful Behavior Scales, Sexual Sensation Seeking Scale, and Marital Aggrandizement Scale were applied. A model with 2 correlated exogenous variables (sexual sensation seeking and marital aggrandizement) and 5 endogenous variables (attitude towards infidelity, marital satisfaction, sexual satisfaction, desire for sexual infidelity, and sexual infidelity behavior) was specified. Six out of 100 participants were dissatisfied with their marriage and 13 with sexual relations with their partner and 20 saw more defects than virtues in their partners. In addition, 83 informed not engaging in sexual infidelity behaviors, 60 indicated total rejection towards own sexual infidelity, 72 showed no desire for sexual infidelity and 51 reported not seeking sexual sensations. The model showed a large effect on the sexual infidelity behavior, explaining $70.8 \%$ of its variance, and had a close fit: $\chi^{2}(7)=11.93$, P-value $=0.10$, RMSEA $=0.03$, and $\mathrm{SRMR}=0.01$. It is concluded that the most critical variable in the model is the desire for sexual infidelity, whose most important determinant was the sexual sensation seeking. The negative correlation between this last variable and marital aggrandizement indicates that these 2 variables can be modulated one to the other. Furthermore, the model highlights aspects of marital and sexual dissatisfaction.

KEYWORDS: married couples, sexual infidelity behavior, desire for sexual infidelity, sexual sensation seeking, path analysis.

*Correspondencia: jose_moral@hotmail.com/Fecha de recepción: 16 de octubre de 2019/Fecha de aceptación: 19 de marzo de $2020 /$ Fecha de publicación: 31 de julio de 2020.

Universidad Autónoma de Nuevo León, Facultad de Psicología, Dr. Carlos Canseco núm. 110, esquina con Dr. Eduardo Aguirre Pequeño, col. Mitras Centro, Monterrey, Nuevo León, México, C. P. 64460. 


\section{INTRODUCCIÓN}

La infidelidad sexual marital puede definirse como el incumplimiento del contrato formal de exclusividad sexual firmado entre los cónyuges. Se suele ocultar a la pareja por las reacciones que pueda generar en la misma, la posibilidad de ruptura marital y el descrédito social (Zapien, 2017). A pesar de sus posibles consecuencias, como la separación o el divorcio, la infidelidad marital es relativamente frecuente (Buss, 2016). En una muestra de 807 mexicanos casados o cohabitantes de los 2 sexos, Moral (2019) reportó un porcentaje de infidelidad sexual del $30.6 \%$ en hombres y $12.8 \%$ en mujeres. El tamaño del efecto del sexo sobre la conducta de infidelidad sexual fue mediano. Ser hombre triplicaba la probabilidad de infidelidad sexual en comparación con ser mujer, lo que se podría atribuir a aspectos socio-biológicos relacionados con una mayor libertad sexual, el concepto cultural de masculinidad y un mayor impulso sexual en los hombres (Lammers y Maner, 2016).

Uno de los temas de interés en la investigación de las parejas casadas es la predicción de la infidelidad sexual (Fisher, 2016; Rosenberg, 2018). En la revisión de estudios planteados desde una perspectiva psicosocial, 5 variables destacan como predictoras de conducta de infidelidad sexual, cuyo efecto se podría contemplar mediado por el deseo de infidelidad sexual. Por una parte, la propia búsqueda de sensaciones sexuales (Kogan y col., 2015) y la actitud positiva hacia la propia infidelidad sexual (Labrecque y Whisman, 2017) pueden incrementar dicho deseo y a través de él aumentar la probabilidad de conducta de infidelidad sexual. Por otra parte, la satisfacción sexual (Scheeren y col., 2018), la satisfacción marital (Rivera-Aragón y col., 2011; Torres y col., 2016), así como la idealización de la pareja y el matrimonio (Starratt y col., 2017) pueden aminorar el deseo y a través de él disminuir la probabilidad de la conducta.

Siguiendo a Romero y col. (2007), se puede distinguir entre conducta manifiesta de infidelidad sexual y conducta encubierta (deseo de in- fidelidad sexual) y plantearse que el deseo es un motivo antecedente a la ejecución de la conducta (Torres y col., 2016). Al ser una conducta ilícita con posibles consecuencias muy negativas, el cumplimiento de este deseo puede ser facilitado por un rasgo de personalidad que implica desinhibición ante estímulos u oportunidades sexuales, como es la búsqueda de sensaciones sexuales (McIntyre y col., 2015).

El objetivo de esta investigación fue desarrollar un modelo predictivo de la conducta sexual infiel en personas casadas, mediante el análisis de siete variables que participan en el fenómeno de la infidelidad.

\section{MATERIALES Y MÉTODOS}

Este estudio empírico tuvo un diseño ex post facto de corte transversal.

\section{Modelo predictivo}

En el modelo se incluyeron 2 variables de personalidad. Por una parte, está la búsqueda de sensaciones sexuales o tendencia a obtener niveles óptimos de excitación sexual e implicarse en experiencias sexuales novedosas (Kalichman y col., 1994). Altos niveles de búsqueda de sensaciones están relacionados con fuerte impulso sexual y problemas de control sexual (McIntyre y col., 2015). Por otra parte, está el engrandecimiento marital o tendencia a exagerar las cualidades positivas de la pareja y minusvalorar o ignorar sus cualidades negativas (O’Rourke y Cappeliez, 2002). El enamoramiento implica un alto nivel de engrandecimiento marital o idealización de la pareja (LópezParra y col., 2018). Se incluyó 1 variable de actitud (hacia la propia infidelidad). Se agregaron 2 variables cognitivas de valoración de la relación: satisfacción marital y satisfacción sexual con la pareja. Finalmente, aparecen 2 variables de infidelidad sexual, una sobre un aspecto motivacional (deseo de infidelidad sexual) y otra sobre un aspecto conductual (haber incurrido en infidelidad sexual).

Se escogieron sólo variables psicosociales con probado efecto directo o indirecto sobre la infidelidad sexual (conducta final) y con un nú- 
mero mínimo para obtener un modelo parsimonioso. Se optó por 2 variables de tendencia o estilo (una sexual y otra de valoración de la relación), 1 de actitud hacia la conducta final, 2 valoraciones subjetivas de la relación (una general y otra específica a la sexualidad) y 1 de deseo hacia la conducta final, partiendo de la revisión de la literatura, en búsqueda de la mayor especificidad y dando primacía al plano subjetivo-motivante. De este modo se logra una propuesta novedosa y parsimoniosa con la que se pretende alcanzar gran poder explicativo y potencial heurístico o explicativo.

El modelo predictivo propuesto se articula en torno a 1 vía no direccional y 14 vías direccionales, cuya justificación se argumenta a continuación:

1. Las variables de personalidad o estructurales son variables no predichas en el modelo (exógenas) y se considera que debe existir una correlación inversa entre las mismas, ya que una alta búsqueda de sensaciones sexuales puede generar un aburrimiento de la pareja Matthews y col., 2018). A su vez, la tendencia a menospreciar a la pareja puede promover la búsqueda de novedades sexuales (ZeiglerHill y col., 2019). Las restantes 5 variables de estado son variables endógenas o predichas en el modelo. Por tanto, se hace una distinción entre estructural (exógeno) y estado o contingente (endógeno), siguiendo la reglas de especificación de modelos (Byrne, 2016).

2. La actitud positiva hacia la propia infidelidad es predicha por una mayor búsqueda de sensaciones sexuales (Matthews y col., 2018) y menor engrandecimiento marital (ZeiglerHill y col., 2019). Las tendencias de comportamiento de ambos rasgos pueden promover una actitud positiva hacia la propia infidelidad, desde un mecanismo de congruencia cognitiva o autojustificación (Jackman, 2015).

3. Una mayor satisfacción marital es predicha por mayor engrandecimiento marital (Gana y col., 2016; Karandashev, 2019) y una ac- titud de mayor rechazo hacia la propia infidelidad marital (Silva y col., 2017), ya que ambas variables facilitan la focalización y valoración positiva de la relación marital (Gana y col., 2016; McNulty y col., 2018).

4. La satisfacción sexual es predicha por mayor satisfacción marital, mayor engrandecimiento marital y mayor búsqueda de sensaciones sexuales con un tamaño del efecto grande (Moral, 2009). Las 2 primeras son variables facilitadoras de una valoración positiva de las relaciones sexuales con el cónyuge (Fugl-Meyer y col., 2019). La tercera puede facilitar el interés por el deseo y la búsqueda de la excitación sexual y el placer con el cónyuge, especialmente en la mujer, al estar su sexualidad socialmente más circunscrita al matrimonio que la del hombre (Shafiee y Etemadi, 2018).

5. El deseo de infidelidad sexual es predicho por menor satisfacción marital (RiveraAragón y col., 2011; Torres y col., 2016), mayor búsqueda de sensaciones sexuales (Kogan y col., 2015), menor engrandecimiento marital (O’Rourke y Cappeliez, 2002; Starratt y col., 2017), mayor aceptación de la propia infidelidad marital (Labrecque y Whisman, 2017) y más insatisfacción sexual con la pareja (Scheeren y col., 2018), ya que son factores motivantes (insatisfacción), facilitadores (actitud) o desinhibidores (búsqueda de sensaciones) de deseo de sexo extradiádico.

6. La conducta de infidelidad sexual es predicha por deseo de infidelidad sexual (Torres y col., 2016) y búsqueda de sensaciones sexuales (Chen y col., 2018). La primera variable actúa como motivante (Buss, 2016) y la segunda como desinhibidor de una conducta socialmente reprobada e incluso ilícita ( $\mathrm{Mc}-$ Intyre y col., 2015).

\section{Participantes}

Los criterios de inclusión fueron: tener entre 18 y 60 años, estar casado con una pareja del sexo opuesto, residir en Monterrey, Nuevo León, saber leer y escribir y proporcionar 
el consentimiento informado. Los criterios de eliminación fueron: cuestionario incompleto $\mathrm{y}$ responder afirmativamente a la pregunta sobre si la pareja estuvo preguntando o mirando las respuestas del cuestionario. Esta pregunta era hecha al recoger el mismo.

Para determinar el tamaño muestral se siguieron las sugerencias para modelamiento de ecuaciones estructurales de Byrne (2016) y Kline (2016). Se pretendía tener aproximadamente 800 casos (400 de cada sexo) y al menos 30 casos por parámetro a estimar. Se colectó una muestra de 820 participantes por el procedimiento de rutas al azar. Se eliminaron 17 casos porque la persona encuestada indicó que la pareja estuvo preguntando. Adicionalmente, se eliminaron 13 casos por datos incompletos.

La muestra final analizada fue de 790 participantes. Se cumplió el mínimo de 30 participantes por parámetro a estimar. Además, se rebasaron los 400 casos en mujeres ( $n=422)$. Aunque el número de hombres quedó por debajo de 400 ( $n=368$ ), logra el porcentaje de los 2 sexos fue estadísticamente equivalente (prueba binomial: $\mathrm{P}=0.059$ exacta bilateral). Por tanto, se satisficieron los criterios fijados para el tamaño muestral. En la Tabla 1 se describen las variables sociodemográficas.

\section{Instrumentos de medida}

Se aplicó un cuestionario de autorreporte impreso. De acuerdo con las normas éticas de investigación de la Asociación Americana de Psicología (APA, por sus siglas en inglés: American Psychological Association) (APA, 2017), el cuestionario iniciaba con un formulario de consentimiento informado en el que se garantizaba el anonimato de las respuestas. A tal fin, no se solicitaron datos de identificación personal. Además, el formulario identificaba al responsable del estudio y proporcionaba su dirección de correo electrónico, para poderlo contactar para cualquier cuestión suscitada por el estudio. A continuación, contenía las preguntas cerradas sobre información sociodemográfica. Finalmente, aparecían
5 instrumentos cuyos ítems tenían una escala de respuesta tipo Likert. Su orden de presentación fue fijo:

Escala de Valoración de la Relación (EVR) (Hendrick y col., 1998). Está integrada por 7 ítems, con 5 categorías ordenadas de respuesta que se puntúan en un rango de 1 a 5 . Una mayor puntuación en la escala refleja mayor satisfacción con la relación (Hendrick y col., 1998). Se empleó la adaptación de la EVR hecha por Moral (2015) en población de parejas mexicanas. En la presente muestra, la consistencia interna fue excelente ( $\alpha$ de Cronbach $=0.90$ y $\alpha$ ordinal $=0.93$ )

Índice de Satisfacción Sexual (ISS) (Hudson, 1992). Está integrado por 25 ítems con 7 categorías ordenadas de respuestas que se puntúan en un rango de 1 a 7 . La redacción de 12 de sus 25 ítems es en sentido de la satisfacción sexual y la de los otros 13 en sentido de la insatisfacción sexual. Conforme a la adaptación de Moral (2018a), hecha en población de parejas mexicanas, se eliminaron 15 ítems (4 de satisfacción y 11 de insatisfacción), resultando el ISS-10. Una mayor puntuación refleja más satisfacción sexual. En la presente muestra la consistencia interna de los 10 ítems fue excelente $(\alpha$ de Cronbach $=$ 0.94 y $\alpha$ ordinal $=0.96$ )

Escala de Actitud hacia la Exclusividad Marital (EAEM) (Weis y Fenton, 1987). Consta de 7 ítems directos, con 5 categorías ordenadas de respuesta que se puntúan en un rango de 1 a 5. Mayor puntuación refleja una actitud más positiva hacia la propia infidelidad. La EAEM no está validada en México. La versión aplicada de la EAEM se obtuvo por traducción reversa. En la presente muestra su consistencia interna fue excelente ( $\alpha$ de Cronbach $=0.94$ y $\alpha$ ordinal $=0.97$ ). Debido a que la EAEM no está validada en la población objeto de estudio, se proporciona información acerca la validez de su modelo de un factor en la muestra. El número de factores fue 1 por el análisis paralelo y la media mínima de las correlaciones parciales al cuadrado. El 
Tabla 1. Descriptivos de las variables sociodemográficas.

Table 1. Descriptions of sociodemographic variables.

\begin{tabular}{|c|c|c|}
\hline Variable & Categorías & $\begin{array}{l}\text { Total } \\
\text { n (\%) }\end{array}$ \\
\hline \multirow{2}{*}{ Sexo } & Mujer & $422(53.4 \%)$ \\
\hline & Hombre & $368(46.6 \%)$ \\
\hline \multirow{5}{*}{ Grado de escolaridad } & Primaria & $26(3.3 \%)$ \\
\hline & Secundaria & $156(19.7 \%)$ \\
\hline & Media superior & $278(35.2 \%)$ \\
\hline & Licenciatura & $286(36.2 \%)$ \\
\hline & Posgrado & $44(5.6 \%)$ \\
\hline \multirow{8}{*}{ Ocupación } & Labores del hogar & $194(24.6 \%)$ \\
\hline & Estudiante & $42(5.3 \%)$ \\
\hline & Desempleado/da & $23(2.9 \%)$ \\
\hline & Jubilado/da & $11(1.4 \%)$ \\
\hline & Empleado/da manual & $100(12.7 \%)$ \\
\hline & Empleado/da técnico/a & $125(15.8 \%)$ \\
\hline & Empleado/da profesional & $237(30 \%)$ \\
\hline & Empresa propia & $58(7.3 \%)$ \\
\hline \multirow{7}{*}{$\begin{array}{l}\text { Ingreso familiar mensual } \\
\text { promedio en el último año } \\
\quad \text { (pesos mexicanos) }\end{array}$} & $<3000$ pesos & $68(8.6 \%)$ \\
\hline & 3000 a 5999 & $212(26.8 \%)$ \\
\hline & 6000 a 11999 & $226(28.6 \%)$ \\
\hline & 12000 a 23999 & $162(20.5 \%)$ \\
\hline & 24000 a 47999 & $75(9.5 \%)$ \\
\hline & 48000 a 91999 & $33(4.2 \%)$ \\
\hline & $\geq 92000$ & $14(1.8 \%)$ \\
\hline \multirow{4}{*}{ Adscripción religiosa } & Cristiana católica & $649(82.2 \%)$ \\
\hline & Cristiana no católica & $72(9.1 \%)$ \\
\hline & Otra & $13(1.6 \%)$ \\
\hline & Ninguna & $56(7.1 \%)$ \\
\hline Variable & & $\begin{array}{c}\text { Total } \\
(\mathrm{M} \pm \mathrm{DE})\end{array}$ \\
\hline Edad & & $34.94 \pm 9.61$ \\
\hline Número total de hijos & & $1.97 \pm 1.30$ \\
\hline Años de estar casados & & $11.26 \pm 8.83$ \\
\hline
\end{tabular}

Estadísticos descriptivos: $\mathrm{M} \pm \mathrm{DE}$ = media aritmética y desviación estándar para muestra, $\mathrm{n}(\%)$ = frecuencia absoluta y porcentaje.

ajuste del modelo de un factor, con siete indicadores y errores de medida independientes por mínimos cuadrados libre de escala, fue bueno, usando como datos de entrada la matriz de correlación policórica: $X^{2} / \mathrm{gl}=36.52 / 14=2.61$, $\mathrm{GFI}=1, \mathrm{AGFI}=0.99, \mathrm{NFI}=1, \mathrm{RFI}=0.99 \mathrm{y}$ $\mathrm{SRMR}=0.04$. Además, el factor mostró validez convergente AVE $=0.81$ y $\omega$ de McDonald $=0.97$. Escalas de Conducta Infiel (ECI) (Romero y col., 2007). Fueron creadas en México. Cuen- tan con 48 ítems directos con 5 categorías ordenadas de respuesta que se puntúan en un rango de 0 a 4 . En el presente estudio no se usó su forma completa de 48 ítems, solo se aplicaron 2 subescalas: la de Conducta de Infidelidad Sexual (CIS), de 21 ítems, y la de Deseo de Infidelidad Sexual (DIS), de 8 items. Debido a que estas dos subescalas fueron extraídas de un inventario más amplio, se validó un modelo de un factor para cada una 
de ellas en la presente muestra. Los 21 ítems de CIS presentaron una consistencia interna excelente ( $\alpha$ de Cronbach $=0.98$ y $\alpha$ ordinal = 0.99). El número de factores de estos 21 ítems convergió en 1 por el análisis paralelo y la media mínima de las correlaciones parciales al cuadrado. El ajuste del modelo de un factor con 21 indicadores y errores de medida independientes fue bueno $\left(X^{2} / \mathrm{gl}=75.99 / 189=0.40, \mathrm{GFI}=1, \mathrm{AGFI}=1\right.$, $\mathrm{NFI}=1, \mathrm{RFI}=1 \mathrm{y} \mathrm{SRMR}=0.02)$; y el factor mostró validez convergente (AVE $=0.87 \mathrm{y}$ $\omega=0.99$ ) por mínimos cuadrados libres de escala, usando como datos de entrada la matriz de correlación policórica. También los 8 ítems de DIS presentaron una consistencia interna excelente ( $\alpha$ de Cronbach $=0.98$ y $\alpha$ or dinal $=0.99)$ y el número de factores de estos 8 ítems convergió en 1 . El ajuste del modelo de un factor con 8 indicadores y errores de medida independientes fue bueno $\left(X^{2} / \mathrm{gl}=\right.$ $2.93 / 20=0.15$, GFI $=1$, AGFI $=1$, NFI $=1$, RFI = 1 y SRMR = 0.01), y el factor tuvo validez convergente (AVE $=0.92$ y $\omega=0.99$ ) usando los mismos métodos.

Escala de Búsqueda de Sensaciones Sexuales (EBSS) (Kalichman y col., 1994). Se compone de 9 ítems directos con 4 categorías ordenadas de respuesta que se puntúan en un rango de 1 a 4. Mayor puntuación refleja mayor tendencia a la búsqueda de excitación, experiencias y novedades sexuales. Se usó la validación en población de parejas mexicanas hecha por Moral (2018b). En la presente muestra su consistencia interna fue buena por el coeficiente alfa de Cronbach $(\alpha$ de Cronbach $=$ 0.89) y excelente por el coeficiente alfa ordinal $(\alpha$ ordinal $=0.93)$.

Escala de Engrandecimiento Marital (EEM) (O' Rourke y Cappeliez, 2002). Consta de 18 ítems con 7 categorías ordenadas de respuesta que se puntúan en un rango de 1 a 7 . Evalúa la distorsión en el sentido de exagerar las cualidades positivas y minimizar las negativas al momento de reportar la satisfacción con la relación de pareja. Se usó la versión en español de Moral (2017) de 14 ítems, desarrollada en población de parejas mexicanas. En esta muestra los 14 ítems presentaron una consistencia interna buena ( $\alpha$ de Cronbach $=0.80 \mathrm{y}$ $\alpha$ ordinal $=0.83$ ).

\section{Procedimientos}

Se seleccionaron de manera aleatoria 82 rutas de una lista de 500 calles en las colonias Mitras Centro, Mitras Norte y Mitras Sur. En cada ruta se escogieron al azar 100 domicilios, para lograr 10 encuestas contestadas. Los números al azar de los domicilios se generaron en una computadora. Se eligieron estas colonias para abarcar un espectro amplio de estratos socioeconómicos y por proximidad espacial. Se pretendía alternar en cada ruta 5 domicilios, en los que contestasen un hombre y una mujer. Los entrevistadores recibieron entrenamiento, por parte del responsable del proyecto de investigación, para colectar los casos y fueron compensados con puntos extras en su calificación en investigación. Hubo una primera fase de colecta de datos, de enero a mayo de 2014 (88 \% de los participantes), y una segunda, de septiembre a noviembre de 2018 (12 \% de los participantes).

\section{Análisis de datos}

Los análisis estadísticos se hicieron con el Paquete estadístico para ciencias sociales (SPSS, por sus siglas en inglés: Statistical Package for the Social Sciences) (IBM SPSS, 2017) versión 24; el paquete para Análisis de estructuras de momentos (AMOS, por sus siglas en inglés: Analysis of Moment Structures) (Arbuckle, 2007) versión 16 y Microsoft Excel versión 2013. Los contrastes fueron a 2 colas, con un nivel de significación de 0.05. El ajuste de las distribuciones de las puntuaciones en las escalas a la normalidad se contrastó por la prueba de Kolmogorov y Smirnov, con la corrección de Lilliefors.

Se contrastó el modelo predictivo por análisis de sendero. Los parámetros se estimaron puntualmente por el método de mínimos cuadrados ponderados (MCP) y por intervalo, con un nivel de confianza al $95 \%$, por el méto- 
do de percentiles corregidos de sesgo (PCS) con la simulación de 2000 muestras aleatorias. Con pesos estructurales estandarizados $(\beta)$ o coeficientes de correlación (r) en valores absolutos menores que 0.10 , se interpretó que los tamaños del efecto o las fuerzas de asociación son triviales; con valores de $|\beta|$ $|\mathrm{r}|$ o entre 0.10 y 0.29 son pequeños; con valores de $|\beta|$ o $|\mathrm{r}|$ entre 0.30 y 0.49 , medios; con valores entre 0.50 y 0.69 son grandes y con valores $\geq 0.70$, muy grandes (Kline, 2016). La proporción de varianza explicada de cada variable endógena por el modelo (correlación múltiple) se calculó a través de la fórmula: $\mathrm{R}^{2}=1-\left(\mathrm{S}^{2}\right.$ (error estructural o de predicción)/ $\mathrm{S}^{2}$ (variable endógena)). Se interpretó que valores de $\mathrm{R}^{2}$ entre 0.02 y 0.129 muestran un tamaño del efecto de los predictores sobre la variable predicha pequeño, entre 0.13 y 0.259 medio, $\mathrm{y} \geq 0.26$ grande (Open Science Collaboration, 2015). Ambos tipos de errores son conceptos estadísticos y corresponden a efectos no atribuibles o desconocidos: en el modelo de medida de los constructos o variables latentes (error de medida) y en el modelo estructural o de predicción (error estructural). Los errores estructurales corresponden a efectos desconocidos no atribuibles al modelo predictivo y difieren de los errores de medida en que estos últimos afectan directamente a las variables.

Siguiendo a Byrne (2016) y Kline (2016), se consideró que el ajuste del modelo a los datos es bueno, al mantenerse la hipótesis nula de bondad de ajuste por la prueba chicuadrado, con una probabilidad > 0.05 en un contraste a 2 colas (P para $\chi^{2}$ ); un valor chicuadrado relativo $\left(\chi^{2} / \mathrm{gl}\right)<2$; una probabilidad > 0.05 por el procedimiento de muestreo repetitivo de Bollen y Stine (1993) (P de BS) con la simulación de 2000 muestras; valores $>0.95$ en el índice de bondad de ajuste (GFI, por sus siglas en inglés: Goodness of Fit Index), índice normado de ajuste (NFI, por sus siglas en inglés: Normed Fit Index), índice incremental de ajuste (IFI, por sus siglas en inglés: Incremental Fit Index) e índice comparativo de ajuste (CFI, por sus siglas en inglés:
Comparative Fit Index); un valor $<0.90$ en el índice de bondad de ajuste corregido (AGFI, por sus siglas en inglés: Adjusted Goodness of Fit Index), así como valores < 0.05 en el error de aproximación cuadrático medio (RMSEA, por sus siglas en inglés: Root Mean Square Error of Approximation) y el residuo estandarizado cuadrático medio (SRMR, por sus siglas en inglés: Standarized Root Mean Square Residual). Se valoró el ajuste como aceptable con P para $\chi^{2}>0.01 ; \chi^{2} / \mathrm{gl}<3$; $\mathrm{P}$ de $\mathrm{BS}>0.01$; GFI, NFI, IFI y CFI > 0.90; AGFI > 0.85; RMSEA $<0.08$ y SRMR $<0.10$.

\section{RESULTADOS}

\section{Distribución y niveles en las 7 variables del modelo predictivo}

Las distribuciones no se ajustaron a la normalidad debido a la presencia de asimetría $(|S k|>0.171=1.960 * \mathrm{EE}$ de $\mathrm{Sk})$ en todas ellas, y desviación de la mesocurtosis $\left(\left|K_{3}\right|>0.341\right.$ $=1.960 * \mathrm{EE}$ de $\mathrm{K}_{3}$ ) en 3 de ellas (EVS, DIS y CIS). Las puntuaciones totales en las escalas se interpretaron en sentido absoluto. Se dividió el rango continuo en el mismo número de intervalos que el número de categorías ordenadas de respuesta a los ítems, usando una amplitud constante: $a=$ (valor máximo - valor mínimo) / número de ítems. De este modo, los contenidos de las categorías ordenadas de respuesta al ítem permitieron interpretar las puntuaciones y los estadísticos de tendencia central de cada variable.

Solo el $5.7 \%$ de los participantes (45 de 790) estaban insatisfechos con su matrimonio (EVR < 2.6), 12.8 \% (101 de 790) estaban insatisfechos con las relaciones sexuales con su pareja (ISS-10 < 3.57) y $20.4 \%$ (161 de 790) veían más defectos que virtudes en sus parejas (EEM-14 < 3.57). El 80.9 \% (639 de 790) reportaron no buscar sensaciones sexuales (EBSS < 2.5), $76.2 \%$ (602 de 790) mostraron una actitud de rechazo hacia la infidelidad sexual (EAEM < 2.6), 72.2 \% (570 de 790) revelaron no tener deseo de infidelidad sexual (DIS < 0.8) y el $83 \%$ (656 de 790) afirmaron no incurrir en conductas de infidelidad sexual $(\mathrm{CIS}<0.8)($ Tabla 2). 
Tabla 2. Estadísticos descriptivos e intervalos para las puntuaciones en las escalas.

Table 2. Descriptive statistics and intervals for scores on the scales.

\begin{tabular}{|c|c|c|c|c|c|c|c|}
\hline Escala & Intervalo & Interpretación & $\mathbf{n}$ & f & $\mathbf{F}$ & Desc1 & ptivos \\
\hline \multirow{4}{*}{ EBSS } & {$[1,1.75)$} & Nada & 404 & 0.511 & 0.511 & M & 1.851 \\
\hline & {$[1.75,2.50)$} & Algo & 235 & 0.298 & 0.809 & $\mathrm{DE}$ & 0.682 \\
\hline & {$[2.50,3.25)$} & Bastante & 135 & 0.171 & 0.980 & Sk & 0.797 \\
\hline & {$[3.25,4]$} & Muchísimo & 16 & 0.020 & 1 & $\mathrm{~K}_{3}$ & 0.011 \\
\hline \multirow{7}{*}{ EEM-14 } & {$[1,1.86)$} & Totalmente en desacuerdo & 9 & 0.011 & 0.011 & $\mathrm{M}$ & 4.428 \\
\hline & {$[1.86,2.71)$} & Bastante en desacuerdo & 48 & 0.061 & 0.072 & Mdn & 4.500 \\
\hline & {$[2.71,3.57)$} & En desacuerdo & 104 & 0.132 & 0.204 & $\mathrm{DE}$ & 1.086 \\
\hline & {$[3.57,4.43)$} & $\mathrm{Ni}$ acuerdo ni en desacuerdo & 230 & 0.291 & 0.495 & Sk & -0.286 \\
\hline & {$[4.43,5.29)$} & De acuerdo & 222 & 0.281 & 0.776 & $\mathrm{~K}_{3}$ & -0.229 \\
\hline & {$[5.29,6.14)$} & Bastante de acuerdo & 148 & 0.187 & 0.963 & & \\
\hline & {$[6.14,7]$} & Totalmente de acuerdo & 29 & 0.037 & 1 & & \\
\hline \multirow{5}{*}{ EAEM } & {$[1,1.80)$} & Total rechazo & 475 & 0.601 & 0.601 & $\mathrm{M}$ & 1.883 \\
\hline & {$[1.80,2.60)$} & Rechazo moderado & 127 & 0.161 & 0.762 & Mdn & 1.286 \\
\hline & {$[2.60,3.40)$} & Ni aceptación ni rechazo & 90 & 0.114 & 0.876 & $\mathrm{DE}$ & 1.112 \\
\hline & {$[3.40,4.20)$} & Aceptación moderada & 49 & 0.062 & 0.938 & Sk & 1.165 \\
\hline & {$[4.20,5]$} & Total aceptación & 49 & 0.062 & 1 & $\mathrm{~K}_{3}$ & 0.311 \\
\hline \multirow{5}{*}{ EVR } & {$[1,1.80)$} & Muy insatisfecho & 6 & 0.008 & 0.008 & M & 3.970 \\
\hline & {$[1.80,2.60)$} & Insatisfecho & 39 & 0.049 & 0.057 & Mdn & 4.143 \\
\hline & {$[2.60,3.40)$} & Ni satisfecho ni insatisfecho & 114 & 0.144 & 0.201 & $\mathrm{DE}$ & 0.737 \\
\hline & {$[3.40,4.20)$} & Satisfecho & 292 & 0.370 & 0.571 & Sk & -0.860 \\
\hline & {$[4.20,5]$} & Muy satisfecho & 339 & 0.429 & 1 & $\mathrm{~K}_{3}$ & 0.578 \\
\hline \multirow{7}{*}{ ISS-10 } & {$[1,1.86)$} & En ningún momento & 16 & 0.020 & 0.020 & M & 5.339 \\
\hline & {$[1.86,2.71)$} & En muy raras ocasiones & 36 & 0.046 & 0.066 & Mdn & 5.800 \\
\hline & {$[2.71,3.57)$} & Algunas veces & 49 & 0.062 & 0.128 & $\mathrm{DE}$ & 1.402 \\
\hline & {$[3.57,4.43)$} & Parte del tiempo & 96 & 0.121 & 0.249 & Sk & -0.878 \\
\hline & {$[4.43,5.29)$} & Buena parte del tiempo & 119 & 0.151 & 0.400 & $\mathrm{~K}_{3}$ & -0.038 \\
\hline & {$[5.29,6.14)$} & La mayor parte del tiempo & 168 & 0.213 & 0.613 & & \\
\hline & {$[6.14,7]$} & En todo momento & 306 & 0.387 & 1 & & \\
\hline \multirow{5}{*}{ DIS } & {$[0,0.80)$} & Nunca & 570 & 0.722 & 0.722 & $\mathrm{M}$ & 0.586 \\
\hline & {$[0.80,1.60)$} & Muy pocas veces & 99 & 0.125 & 0.847 & Mdn & 0 \\
\hline & {$[1.60,2.40)$} & Pocas veces & 74 & 0.094 & 0.941 & $\mathrm{DE}$ & 0.911 \\
\hline & {$[2.40,3.20)$} & Frecuentemente & 32 & 0.040 & 0.981 & Sk & 1.732 \\
\hline & {$[3.20,4]$} & Casi siempre & 15 & 0.019 & 1 & $\mathrm{~K}_{3}$ & 2.408 \\
\hline \multirow{5}{*}{ CIS } & {$[0,0.80)$} & Nunca & 656 & 0.830 & 0.830 & M & 0.363 \\
\hline & {$[0.80,1.60)$} & Muy pocas veces & 60 & 0.076 & 0.906 & Mdn & 0 \\
\hline & {$[1.60,2.40)$} & Pocas veces & 51 & 0.065 & 0.971 & $\mathrm{DE}$ & 0.693 \\
\hline & {$[2.40,3.20)$} & Frecuentemente & 21 & 0.026 & 0.997 & Sk & 2.267 \\
\hline & {$[3.20,4]$} & Casi siempre & 2 & 0.003 & 1 & $\mathrm{~K}_{3}$ & 4.615 \\
\hline
\end{tabular}

Escalas: EBSS = Búsqueda de Sensaciones Sexuales, EEM-14 = Engrandecimiento Marital, EAEM = Actitud hacia la Exclusividad Marital, EVR = Valoración de la Relación, ISS-10 = Satisfacción Sexual, DIS = Deseo de Infidelidad Sexual, y CIS $=$ Conducta de Infidelidad Sexual. $n=$ frecuencia absoluta, $\mathrm{f}=$ frecuencia relativa, $\mathrm{F}=$ frecuencia acumulada, $\mathrm{M}=$ media, $\mathrm{Mdn}$ = mediana, $\mathrm{DE}$ = desviación estándar, $\mathrm{Sk}=$ coeficiente de asimetría, $\mathrm{K}_{3}=$ exceso de curtosis. Errores estándar de $\mathrm{Sk}=0.087$ y de $\mathrm{K}_{3}=0.174$ en las 7 variables. Tamaño muestral: $N=790$. 
Modelo predictivo de conducta de infidelidad sexual

Se especificó el modelo recursivo de acuerdo a las vías asociativas y direccionales enunciadas en la sección de Materiales y Métodos. El peso de la satisfacción marital sobre el deseo de infidelidad sexual no fue significativo, tanto por MCP ( $\mathrm{B}=-0.097$, intervalo de confianza [IC] al $95 \%$ (- 0.211, 0.017), $\mathrm{Z}=-1.672, \mathrm{P}=0.091)$ como por $\mathrm{PCS}(\beta=-0.079$, IC al $95 \%$ (- 0.200, 0.012), $P=0.082)$. Tras eliminar esta vía no significativa se estimó de nuevo el modelo. Todos los parámetros (pesos estructurales o efectos directos, varianzas y covarianzas) fueron significativos por los métodos MCP y PCS (Figura 1). El ajuste fue bueno por todos los índices (Tabla 3).
La correlación entre búsqueda de sensaciones sexuales y engrandecimiento marital fue negativa y con una fuerza de asociación pequeña, $r=-0.189$, IC al $95 \%$ (- 0.262, - 0.113). Al elevar al cuadrado esta correlación y multiplicar por 100 se obtiene que la varianza compartida por las dos variables exógenas fue del $3.6 \%$ (Tabla 4).

El modelo explicó el $30 \%$ de la varianza de actitud positiva hacia la propia infidelidad:

$\mathrm{R}^{2}=1-\frac{\mathrm{S}_{\mathrm{e} 1}^{2}}{\mathrm{~S}_{\mathrm{API}}^{2}}=0.300, \mathrm{IC}$ al $95 \%(0.237,0.364)$

(Tabla 4). El efecto de la búsqueda de sensaciones sexuales fue directo, positivo y de tamaño

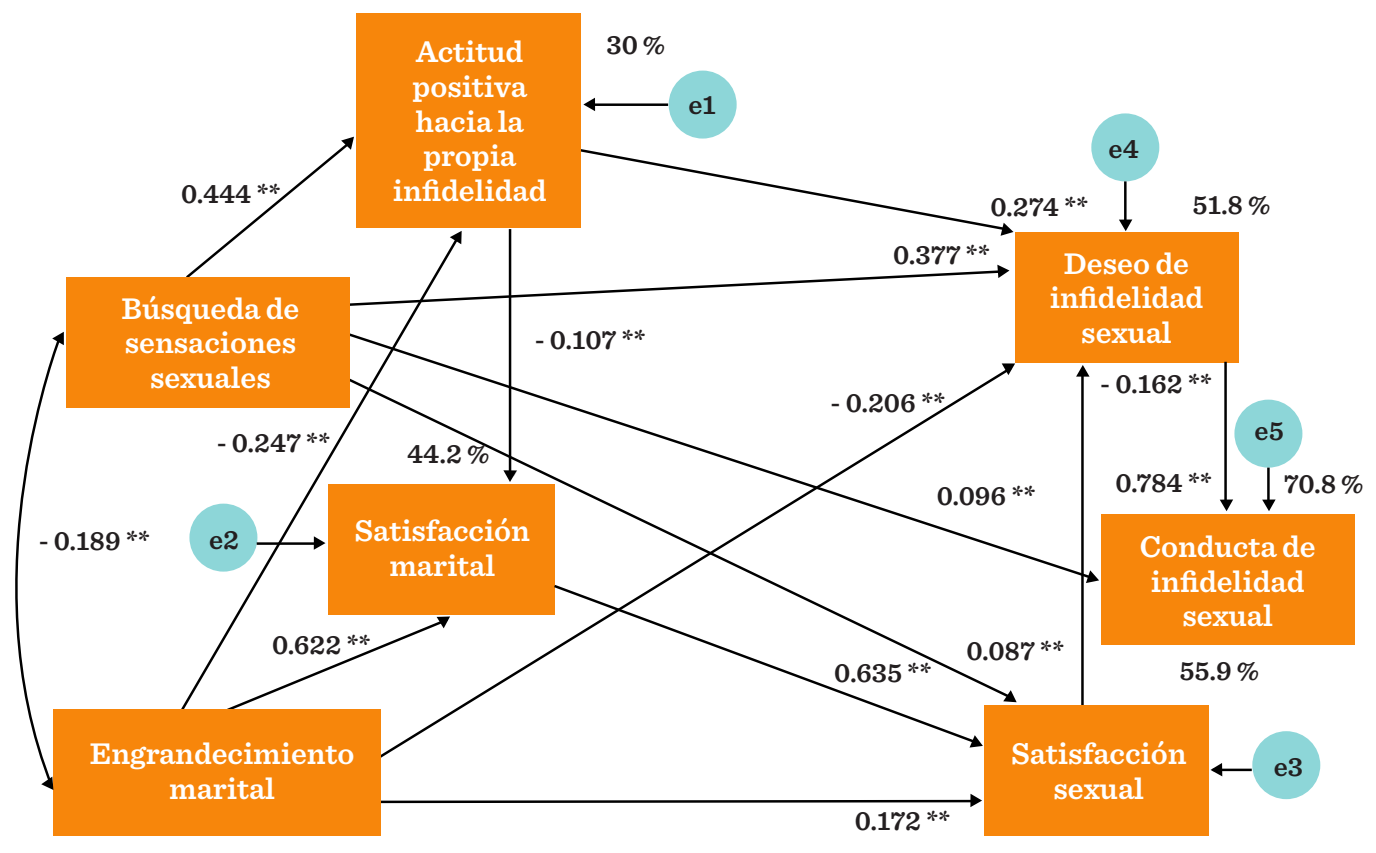

Figura 1. Modelo hipotético modificado (sin el efecto directo de la satisfacción marital sobre deseo de infidelidad sexual) en un análisis unigrupo en la muestra conjunta de 790 participantes. Los parámetros se estimaron por Mínimos Cuadrados Ponderados y su significación se contrastó por Percentiles Corregidos de Sesgo con la simulación de 2000 muestras aleatorias: ${ }^{\text {ns }} \mathrm{P}>0.05$, ${ }^{*} \mathrm{P} \leq 0.05,{ }^{* *} \mathrm{P} \leq 0.01$. En círculos se señalan las 5 variables exógenas latentes del modelo que corresponden a los errores estructurales (e1 a e5).

Figure 1. Modified hypothetical model (without the direct effect of marital satisfaction on desire for sexual infidelity) in a one-group analysis in the pooled sample of 790 participants. The parameters were estimated by Weighted Least Squares and their significance was tested by Corrected-Bias Percentiles with the simulation of 2000 random samples: ${ }^{\text {ns }} \mathrm{P}>0.05$, ${ }^{*} \mathrm{P} \leq 0.05,{ }^{* *} \mathrm{P} \leq 0.01$. The 5 latent exogenous variables of the model, that correspond to the structural errors, are indicated in circles (e1 to e5). 
Tabla 3. Índices de ajuste del modelo modificado.

Table 3. Fit indices of the modified model.

\begin{tabular}{|c|c|c|c|}
\hline \multirow{2}{*}{$\begin{array}{c}\text { Indices } \\
\text { de ajuste }\end{array}$} & Bueno & Aceptable & Valores \\
\cline { 2 - 4 } & & & 11.925 \\
\hline$X^{2}$ & & & 7 \\
\hline gl & $>0.05$ & $>0.01$ & 0.103 \\
\hline $\mathrm{P}$ & $\leq 2$ & $\leq 3$ & 1.704 \\
\hline$X^{2} / \mathrm{gl}$ & $>0.05$ & $>0.01$ & 0.128 \\
\hline P de BS & $\geq 0.95$ & $\geq 0.90$ & 0.992 \\
\hline GFI & $\geq 0.90$ & $\geq 0.85$ & 0.970 \\
\hline AGFI & $\geq 0.95$ & $\geq 0.90$ & 0.974 \\
\hline NFI & $\geq 0.95$ & $\geq 0.90$ & 0.989 \\
\hline IFI & $\geq 0.95$ & $\geq 0.90$ & 0.989 \\
\hline CFI & $\leq 0.05$ & $\leq 0.075$ & 0.030 \\
\hline RMSEA & $>0.10$ & $>0.05$ & 0.866 \\
\hline P-close & $\leq 0.05$ & $<0.10$ & 0.014 \\
\hline SRMR & & & \\
\hline
\end{tabular}

Modelo modificado, sin el efecto directo de la satisfacción marital sobre deseo de infidelidad sexual. Fue estimado por mínimos cuadrados ponderados. Índices de ajuste: $\chi^{2}=$ prueba chi-cuadrado, $\mathrm{gl}=$ grados de libertad, $\mathrm{P}$ de $\mathrm{BS}=$ probabilidad para un contraste a 2 colas, $\chi^{2} / \mathrm{gl}=$ chi-cuadrado relativa, $\mathrm{P}$ de $\mathrm{BS}=$ probabilidad por el procedimiento de muestreo repetitivo de Bollen y Stine (1993), con la simulación de 2000 muestras aleatorias, GFI = índice de bondad de ajuste, AGFI = índice de bondad de ajuste corregido, NFI = índice normado de ajuste, IFI = índice incremental de ajuste, $\mathrm{CFI}=$ índice comparativo de ajuste, RMSEA = error de aproximación cuadrático medio, $\mathrm{P}$-close $=$ probabilidad de la hipótesis nula Ho: RMSEA $\leq 0.05 \mathrm{y}$ SRMR = residuo estandarizado cuadrático medio. Tamaño muestral: $\mathrm{N}=790$.

medio: $\beta$ total $=\beta$ directo $=0.444, \mathrm{IC}$ al $95 \%$ (0.376, 0.500). El efecto del engrandecimiento marital también fue directo, pero negativo y de tamaño pequeño: $\beta$ total $=\beta$ directo $=-0.247, \mathrm{IC}$ al $95 \%$ (- 0.309, - 0.184) (Figura 1 y Tabla 5).

El modelo explicó el $44.2 \%$ de la varianza de satisfacción marital:

$\mathrm{R}^{2}=1-\frac{\mathrm{S}_{\mathrm{e} 2}^{2}}{\mathrm{~S}_{\mathrm{SM}}^{2}}=0.442, \mathrm{IC}$ al $95 \%(0.391,0.493)$

(Tabla 4). El efecto directo del engrandecimiento marital fue positivo y de tamaño grande: $\beta$ directo $=0.622$, IC al $95 \%(0.575,0.667)$. Además, su efecto indirecto, a través de la actitud positiva hacia la propia infidelidad, también fue significativo y del mismo signo: Bindirecto $=0.027$, IC al $95 \%(0.012,0.047)$. Consecuentemente, su efecto total aumentó: $\beta$ total $=0.648$, IC al $95 \%$ (0.607, 0.687). El efecto de la actitud positiva hacia la propia infidelidad fue directo, negativo y de tamaño pequeño: $\beta$ total $=\beta$ directo $=-0.107, \mathrm{IC}$ al $95 \%$ (- 0.173, - 0.046). A su vez, la búsqueda de sensaciones sexuales tuvo un efecto indirecto mediado por actitud positiva hacia la propia infidelidad; su signo fue negativo $\mathrm{y}$ su tamaño trivial: $\beta$ indirecto $=-0.048$, IC al $95 \%$ (- 0.079, - 0.020) (Figura 1 y Tabla 5).

El modelo explicó el $55.9 \%$ de la varianza de la satisfacción sexual:

$R^{2}=1-\frac{S_{\mathrm{e} 3}^{2}}{S_{\mathrm{SS}}^{2}}=0.559, \mathrm{IC}$ al $95 \%(0.489,0.615)$

(Tabla 4). La satisfacción marital tuvo un efecto directo, positivo y de tamaño grande: $\beta$ total $=\beta$ directo $=0.635, \mathrm{IC} 95 \%(0.563,0.696) . \mathrm{El}$ efecto directo y positivo del engrandecimien- 
Tabla 4. Correlación entre las dos variables exógenas, varianzas de los residuos y las variables endógenas, y correlación múltiple o varianza explicada de cada variable endógena.

Table 4. Correlation between the two exogenous variables, variances of the residuals and endo-genous variables, and multiple correlation or explained variance of each endogenous variable.

\begin{tabular}{|c|c|c|c|c|c|}
\hline $\begin{array}{c}\text { Variables } \\
\text { en el modelo }\end{array}$ & Relación & $\begin{array}{c}r \\
(I C 95 \%)\end{array}$ & $\begin{array}{c}\mathbf{S}^{2} \text { ei } \\
\text { (IC 95\%) }\end{array}$ & $\begin{array}{c}\mathbf{S}^{2} \mathbf{X i} \\
(\mathbf{I C} 95 \%)\end{array}$ & $\begin{array}{c}\mathbf{R}^{2} \\
(\mathbf{I C ~ 9 5 \% )}\end{array}$ \\
\hline & No direccional & & & & \\
\hline \multirow[t]{2}{*}{$\begin{array}{l}\text { Variables } \\
\text { endógenas }\end{array}$} & BSS y EM & $\begin{array}{c}-0.189 \\
(-0.262,-0.113)\end{array}$ & & & \\
\hline & Direccional: ei $\bullet \mathrm{Xi}$ & & & & \\
\hline \multirow{5}{*}{$\begin{array}{l}\text { Variables } \\
\text { exógenas }\end{array}$} & $\mathrm{e} 1 \triangleright \mathrm{API}$ & & $\begin{array}{c}0.864 \\
(0.769,0.990)\end{array}$ & $\begin{array}{c}1.235 \\
(1.113,1.368)\end{array}$ & $\begin{array}{c}0.300 \\
(0.237,0.364)\end{array}$ \\
\hline & $\mathrm{e} 2 \triangleright \mathrm{SM}$ & & $\begin{array}{c}0.303 \\
(0.274,0.345)\end{array}$ & $\begin{array}{c}0.543 \\
(0.484,0.603)\end{array}$ & $\begin{array}{c}0.442 \\
(0.391,0.493)\end{array}$ \\
\hline & $\mathrm{e} 3 \bullet \mathrm{SS}$ & & $\begin{array}{c}0.865 \\
(0.750,1.020)\end{array}$ & $\begin{array}{c}1.962 \\
(1.790,2.151)\end{array}$ & $\begin{array}{c}0.559 \\
(0.489,0.615)\end{array}$ \\
\hline & $\mathrm{e} 4 \bullet \mathrm{DIS}$ & & $\begin{array}{c}0.399 \\
(0.339,0.490)\end{array}$ & $\begin{array}{c}0.828 \\
(0.716,0.956)\end{array}$ & $\begin{array}{c}0.518 \\
(0.440,0.580)\end{array}$ \\
\hline & e $5 \triangleright$ CIS & & $\begin{array}{c}0.140 \\
(0.119,0.205)\end{array}$ & $\begin{array}{c}0.479 \\
(0.396,0.570)\end{array}$ & $\begin{array}{c}0.708 \\
(0.616,0.762)\end{array}$ \\
\hline
\end{tabular}

Estadísticos: r (IC $95 \%$ ) = estimación puntual y por intervalo, con un nivel de confianza al $95 \%$ de la correlación entre las dos variables exógenas por el coeficiente de correlación producto-momento de Pearson; $\mathrm{S}^{2}$ ei (IC $95 \%$ ) = estimación puntual y por intervalo con un nivel de confianza al $95 \%$ de la varianza de los errores estructurales o de predicción (efectos no atribuibles o desconocidos que influyen en la variable predicha, S²xi (IC $95 \%$ ) = estimación puntual y por intervalo, con un nivel de confianza al $95 \%$ de la varianza de las variables endógenas; $\mathrm{R}^{2}(\mathrm{IC} 95 \%)$ e estimación puntual y por intervalo, con un nivel de confianza al $95 \%$ de la correlación múltiple o varianza explicada de cada variable endógena. Las estimaciones puntuales de las correlaciones y las varianzas de los errores estructurales o de predicción se hicieron por mínimos cuadrados ponderados y la estimación por intervalo por percentiles corregidos de sesgo con la simulación de 2000 muestras. Las estimaciones puntuales de las varianzas de las variables exógenas (BSS, EM, e1, e2, e3, e4 y e5) corresponden a varianzas muestrales, y las estimaciones por intervalo se hicieron por percentiles corregidos de sesgo y acelerados con la simulación de 2000 muestras. Variables: BSS = búsqueda de sensaciones sexuales; EM = engrandecimiento marital; API = actitud positiva hacia la infidelidad marital; $\mathrm{SM}=$ satisfacción marital; DIS = deseo de infidelidad sexual; $\mathrm{SS}=$ satisfacción sexual; y CIS = conducta de infidelidad sexual.

to marital fue pequeño: $\beta$ directo $=0.172, \mathrm{IC}$ al $95 \%(0.109,0.239)$. Además, su efecto indirecto vía actitud-satisfacción marital fue medio y del mismo signo: $\beta$ indirecto $=0.412$, $\mathrm{IC}$ al $95 \%$ (0.361, 0.459). Consecuentemente, la suma de estos dos efectos resultó en un efecto total grande: $\beta$ total $=0.584$, IC al $95 \%$ (0.531, 0.635). La búsqueda de sensaciones sexuales tuvo efecto directo, positivo y de tamaño trivial: $\beta$ directo $=0.087$, IC al $95 \%$ (0.029, 0.136). Su efecto indirecto vía actitud-satisfacción marital también fue significativo y trivial, pero de signo opuesto: $\beta$ indirecto $=-0.030$, IC al $95 \%$ (- 0.051, - 0.012). Consecuentemente, estos 2 tamaños de efecto trivial y de signo opuesto resul- taron en un efecto total no significativo: $\beta$ total $=0.056$, IC al $95 \%$ (- 0.007, 0.110). No obstante, el peso de esta variable (efecto directo) fue significativo por ambos métodos $(\mathrm{B}=0.17 \%$, IC $95 \%$ (0.074, 0.280), $\mathrm{Z}=3.376, \mathrm{P}<0.001$ por MCP; у $\beta=0.087$, IC $95 \%$ (0.029, 0.136), $\mathrm{P}=0.002$ por PCS). Al eliminar esta vía, empeoró significativamente el ajuste $\left(\chi^{2}[1]=11.344\right.$, $P=0.001)$, la bondad de ajuste ya no se mantuvo por la prueba chi-cuadrado $\left(\chi^{2}[8]=23.269\right.$, $\mathrm{P}=0.003)$ ni por la probabilidad de muestreo repetitivo ( $\mathrm{P}$ de $\mathrm{BS}=0.005$ ), la chi-cuadrada relativa fue mayor que $2\left(\chi^{2} / \mathrm{gl}=2.909\right)$ y $\operatorname{los}$ índices de mejora de ajuste sugerían su inclusión; por lo que se conservó en el modelo. 
Tabla 5. Valores estandarizados de los efectos directos, indirectos y totales en el modelo modificado. Table 5. Standardized values of the direct, indirect and total effects in the modified model.

\begin{tabular}{|c|c|c|c|c|c|c|c|}
\hline \multirow{2}{*}{ Vía } & \multicolumn{2}{|c|}{ Efecto directo } & \multicolumn{2}{|c|}{ Efecto indirecto } & \multicolumn{3}{|c|}{ Efecto total } \\
\hline & $\beta_{\mathbf{d}}$ & $\mathbf{P}$ & Mediado & $\beta_{i}$ & $\mathbf{P}$ & $\beta_{t}$ & $\mathbf{P}$ \\
\hline $\mathrm{BSS} \bullet \mathrm{API}$ & $\begin{array}{c}0.444 \\
(0.376,0.500)\end{array}$ & 0.002 & & & & $\begin{array}{c}0.444 \\
(0.376,0.500)\end{array}$ & 0.002 \\
\hline $\mathrm{EM} \bullet \mathrm{API}$ & $\begin{array}{c}-0.247 \\
(-0.309,-0.184)\end{array}$ & 0.001 & & & & $\begin{array}{c}-0.247 \\
(-0.309,-0.184)\end{array}$ & 0.001 \\
\hline $\mathrm{API} \triangleright \mathrm{SM}$ & $\begin{array}{c}-0.107 \\
(-0.173,-0.046)\end{array}$ & 0.002 & & & & $\begin{array}{c}-0.107 \\
(-0.173,-0.046)\end{array}$ & 0.002 \\
\hline $\mathrm{BSS} \bullet \mathrm{SM}$ & & & API & $\begin{array}{c}-0.048 \\
(-0.079,-0.020)\end{array}$ & 0.002 & $\begin{array}{c}-0.048 \\
(-0.079,-0.020)\end{array}$ & 0.002 \\
\hline $\mathrm{EM} \bullet \mathrm{SM}$ & $\begin{array}{c}0.622 \\
(0.575,0.667)\end{array}$ & 0.001 & API & $\begin{array}{c}0.027 \\
(0.012,0.047)\end{array}$ & 0.001 & $\begin{array}{c}0.648 \\
(0.607,0.687)\end{array}$ & 0.001 \\
\hline $\mathrm{API} \triangleright \mathrm{SS}$ & & & SM & $\begin{array}{c}-0.068 \\
(-0.112,-0.028)\end{array}$ & 0.002 & $\begin{array}{c}-0.068 \\
(-0.112,-0.028)\end{array}$ & 0.002 \\
\hline $\mathrm{BSS} \bullet \mathrm{SS}$ & $\begin{array}{c}0.087 \\
(0.029,0.136)\end{array}$ & 0.002 & $\mathrm{API} \triangleright \mathrm{SM}$ & $\begin{array}{c}-0.030 \\
(-0.051,-0.012)\end{array}$ & 0.002 & $\begin{array}{c}0.056 \\
(-0.007,0.110)\end{array}$ & 0.087 \\
\hline $\mathrm{SM} \bullet \mathrm{SS}$ & $\begin{array}{c}0.635 \\
(0.563,0.696)\end{array}$ & 0.002 & & & & $\begin{array}{c}0.635 \\
(0.563,0.696)\end{array}$ & 0.002 \\
\hline $\mathrm{EM} \bullet \mathrm{SS}$ & $\begin{array}{c}0.172 \\
(0.109,0.239)\end{array}$ & 0.001 & $\mathrm{API} \vee \mathrm{SM}$ & $\begin{array}{c}0.412 \\
(0.361,0.459)\end{array}$ & 0.002 & $\begin{array}{c}0.584 \\
(0.531,0.635)\end{array}$ & 0.001 \\
\hline $\mathrm{API} \bullet \mathrm{DIS}$ & $\begin{array}{c}0.274 \\
(0.192,0.359)\end{array}$ & 0.001 & $\mathrm{SM} \bullet \mathrm{SS}$ & $\begin{array}{c}0.011 \\
(0.004,0.022)\end{array}$ & 0.001 & $\begin{array}{c}0.285 \\
(0.202,0.371)\end{array}$ & 0.001 \\
\hline $\mathrm{BSS} \bullet \mathrm{DIS}$ & $\begin{array}{c}0.377 \\
(0.301,0.443)\end{array}$ & 0.002 & $\begin{array}{l}\text { 1) } \mathrm{API} \\
\text { 2) } \mathrm{SS}\end{array}$ & $\begin{array}{c}0.113 \\
(0.072,0.60)\end{array}$ & 0.001 & $\begin{array}{c}0.489 \\
(0.428,0.544)\end{array}$ & 0.002 \\
\hline EM•DIS & $\begin{array}{c}-0.206 \\
(-0.270,-0.149)\end{array}$ & 0.001 & $\begin{array}{l}\text { 1) } \mathrm{API} \\
\text { 2) } \mathrm{SS}\end{array}$ & $\begin{array}{c}-0.162 \\
(-0.210,-0.117)\end{array}$ & 0.001 & $\begin{array}{c}-0.368 \\
(-0.420,-0.312)\end{array}$ & 0.001 \\
\hline $\mathrm{SM} \bullet \mathrm{DIS}$ & & & SS & $\begin{array}{c}-0.103 \\
(-0.147,-0.061)\end{array}$ & 0.001 & $\begin{array}{c}-0.103 \\
(-0.147,-0.061)\end{array}$ & 0.001 \\
\hline $\mathrm{SS} \triangleright \mathrm{DIS}$ & $\begin{array}{c}-0.162 \\
(-0.228,-0.095)\end{array}$ & 0.001 & & & & $\begin{array}{c}-0.162 \\
(-0.228,-0.095)\end{array}$ & 0.001 \\
\hline $\mathrm{API} \triangleright \mathrm{CIS}$ & & & $\begin{array}{l}\text { 1) DIS } \\
\text { 2) } S M \bullet S S \bullet D I S\end{array}$ & $\begin{array}{c}0.223 \\
(0.154,0.291)\end{array}$ & 0.002 & $\begin{array}{c}0.223 \\
(0.154,0.291)\end{array}$ & 0.002 \\
\hline $\mathrm{BSS} \bullet \mathrm{CIS}$ & $\begin{array}{c}0.096 \\
(0.043,0.172)\end{array}$ & 0.002 & $\begin{array}{l}\text { 1) DIS } \\
\text { 2) } \mathrm{SS} \bullet \mathrm{DIS}\end{array}$ & $\begin{array}{c}0.383 \\
(0.312,0.437)\end{array}$ & 0.006 & $\begin{array}{c}0.480 \\
(0.418,0.542)\end{array}$ & 0.001 \\
\hline $\mathrm{SM} \bullet \mathrm{CIS}$ & & & $\mathrm{SS} \bullet \mathrm{DIS}$ & $\begin{array}{c}-0.080 \\
(-0.117,-0.047)\end{array}$ & 0.001 & $\begin{array}{c}-0.080 \\
(-0.117,-0.047)\end{array}$ & 0.001 \\
\hline EM $•$ CIS & & & $\begin{array}{l}\text { 1) DIS } \\
\text { 2) SS DIS } \\
\text { 3) API DIS }\end{array}$ & $\begin{array}{c}-0.289 \\
(-0.334,-0.239)\end{array}$ & 0.003 & $\begin{array}{c}-0.289 \\
(-0.334,-0.239)\end{array}$ & 0.003 \\
\hline $\mathrm{SS} \triangleright \mathrm{CIS}$ & & & DIS & $\begin{array}{c}-0.127 \\
(-0.182,-0.074)\end{array}$ & 0.002 & $\begin{array}{c}-0.127 \\
(-0.182,-0.074)\end{array}$ & 0.002 \\
\hline DIS $• \mathrm{CIS}$ & $\begin{array}{c}0.784 \\
(0.695,0.837)\end{array}$ & 0.013 & & & & $\begin{array}{c}0.784 \\
(0.695,0.837) \\
\end{array}$ & 0.013 \\
\hline
\end{tabular}

Modelo modificado sin el efecto directo de satisfacción marital sobre deseo de infidelidad sexual en la muestra de 790 participantes. Estimación puntual por mínimos cuadrados ponderados y estimación por intervalo con un nivel de confianza al $95 \%$ y probabilidad en un contraste a 2 colas por percentiles corregidos de sesgo. $\quad$ = vía direccional, $\beta \mathrm{d}$ = peso estructural estandarizado del efecto directo (de la variable $\mathrm{x}$ sobre y) que son los valores que aparecen en la Figura 1, $\beta \mathrm{i}$ = peso estructural estandarizado del efecto indirecto de la variable $\mathrm{x}$ sobre y mediado por las variables o vías y $\beta \mathrm{t}=$ peso estructural estandarizado del efecto total o suma de ambos efectos, $\mathrm{P}=$ valor de probabilidad en un contraste a 2 colas bajo la hipótesis nula de efecto nulo. Variables exógenas: BSS = búsqueda de sensaciones sexuales y EM = engrandecimiento marital. Variables endógenas: API = actitud positiva hacia la infidelidad marital, SM = satisfacción marital, SS = satisfacción sexual, DIS = deseo de infidelidad sexual y CIS = conducta de infidelidad sexual. 
Finalmente, la actitud positiva hacia la propia infidelidad tuvo un efecto indirecto, vía satisfacción marital-búsqueda de sensaciones sexuales de signo negativo y tamaño trivial: $\beta$ total $=\beta$ directo $=-0.068, \mathrm{IC}$ al $95 \%(-0.112,-0.028)$ (Figura 1 y Tabla 5).

El modelo explicó el $51.8 \%$ de la varianza del deseo de infidelidad sexual:

$\mathrm{R}^{2}=1-\frac{\mathrm{S}_{\mathrm{e} 4}^{2}}{\mathrm{~S}_{\mathrm{DIS}}^{2}}=0.518, \mathrm{IC}$ al $95 \%(0.440,0.580)$

(Tabla 4). La búsqueda de sensaciones sexuales tuvo un efecto directo, positivo y de tamaño medio: $\beta$ directo $=0.37 \%$, IC al $95 \%$ (0.301, 0.443). $\mathrm{El}$ efecto directo de la actitud positiva hacia la propia infidelidad fue de tamaño pequeño y signo positivo: $\beta$ directo $=0.274$, IC al $95 \%$ (0.192, 0.359). Los efectos directos del engrandecimiento marital y la satisfacción sexual también fueron de tamaño pequeño, pero de signo negativo: $\beta$ directo $=-0.206$, IC al $95 \%$ $(-0.270,-0.149)$ y $\beta$ directo $=-0.162, \mathrm{IC}$ al $95 \%$ (- 0.228, - 0.095), respectivamente. A su vez, los 4 efectos indirectos, mediados por la satisfacción sexual, fueron significativos. El de la búsqueda de sensaciones sexuales fue positivo y pequeño: $\beta$ indirecto $=0.113$, IC al $95 \%$ (0.072, 0.160). Los del engrandecimiento y satisfacción maritales fueron negativos y pequeños: $\beta$ indirecto $=-0.162$, IC al $95 \%$ (- 0.210, - 0.117) y $\beta$ indirecto $=-0.103$, IC al $95 \%$ (- 0.147, - 0.061), respectivamente. El de la actitud positiva hacia la infidelidad fue positivo y trivial: $\beta$ indirecto $=0.011, \mathrm{IC}$ al $95 \%(0.004,0.022)$ (Figura 1 y Tabla 5).

El modelo explicó el $70.8 \%$ de la varianza de la conducta de infidelidad sexual:

$\mathrm{R}^{2}=1-\frac{\mathrm{S}_{\mathrm{e} 5}^{2}}{\mathrm{~S}_{\mathrm{CIS}}^{2}}=0.708, \mathrm{IC}$ al $95 \%(0.616,0.762)$

(Tabla 4). El efecto del deseo de infidelidad sexual sobre la conducta de infidelidad sexual fue directo, positivo y de tamaño muy grande: $\beta$ total $=\beta$ directo $=0.784, \mathrm{IC}$ al $95 \%$ (0.695, 0.837). La búsqueda de sensaciones se- xuales tuvo un efecto directo, positivo y de tamaño trivial: $\beta$ directo $=0.096$, IC al $95 \%$ (0.043, 0.172). No obstante, su efecto indirecto fue medio y del mismo signo: $\beta$ indirecto $=0.383$, IC al $95 \%(0.312,0.437)$. Como consecuencia, la suma de ambos efectos dio un efecto total medio: 0.480, IC al $95 \%$ (0.418, 0.542). A su vez, los 4 efectos indirectos restantes, mediados por el deseo de infidelidad sexual, fueron significativos. El de actitud positiva hacia la propia infidelidad tuvo signo positivo: $\beta$ indirecto $=0.223, \mathrm{IC}$ al $95 \%$ (0.154, 0.291). Los del engrandecimiento marital y satisfacción sexual tuvieron signos negativos: $\beta$ indirecto $=-0.289$, IC al $95 \%(-0.334,-0.239)$ y $\beta$ indirecto $=-0.127$, IC al $95 \%(-0.182,-0.074)$, respectivamente. Los tres fueron de tamaño pequeño. El de satisfacción marital fue negativo y trivial: $\beta$ indirecto $=-0.080, \mathrm{IC}$ al $95 \%$ (- 0.117, - 0.047) (Figura 1 y Tabla 5).

\section{DISCUSIÓN}

Diversas investigaciones hechas en población general o no clínica revelan asimetría negativa (casos atípicos en los valores bajos) y niveles altos de satisfacción marital, satisfacción sexual y engrandecimiento marital (O’Rourke y Cappeliez, 2002; Moral, 2009; Yucel y Koydemir, 2015; Fallis y col., 2016); asimismo, asimetría positiva (casos atípicos en los valores altos) y niveles bajos en búsqueda de sensaciones sexuales (Weinstein y col., 2015), actitud positiva hacia la propia infidelidad (Labrecque y Whisman, 2017), así como en deseo de infidelidad sexual y conducta de infidelidad sexual. Estos sesgos se atribuyen a que son personas casadas comprometidas con su familia y, en la mayoría de los casos, sin problemas de desamor o conflictos insuperables (Rivera-Aragón y col., 2011; Torres y col., 2016). No obstante, las puntuaciones en búsqueda de sensaciones sexuales pueden aproximarse más a una distribución normal al tratarse de un rasgo de personalidad temperamental (Norbury y col., 2015).

De acuerdo con lo previamente señalado, las puntuaciones en satisfacción marital (EVR) de Hendrick y col. (1998), satisfacción sexual 
(ISS-10) de Moral (2018a) y engrandecimiento marital (EEM-14) de Moral (2017) se concentraron en los valores altos, y los casos atípicos surgieron en la cola izquierda, evidenciando que el estilo valorativo de idealización de la pareja y las valoraciones positivas de la relación dominan, conforme a la expectativa (O’Rourke y Cappeliez, 2002; Moral, 2009; Yucel y Koydemir, 2015; Fallis y col., 2016). Este tipo de asimetría se puede atribuir a la naturaleza de la población de la cual se extrajo la muestra. En población general, la mayoría tiene un matrimonio funcional y estable, en contraste con las personas que acuden a terapia de pareja o familiar (Stanford, 2016; Rosenberg, 2018). También se cumplió la expectativa de concentración de las puntuaciones en los valores bajos y cola larga hacia la derecha en las subescalas de conducta de infidelidad sexual (CIS) y deseo de infidelidad sexual (DIS) extraídas del ECI de Romero y col. (2007), actitud hacia la propia infidelidad sexual (EAEM) de Weis y Fenton (1987) y búsqueda de sensaciones sexuales (EBSS) de Kalichman y col. (1994). Esta asimetría se mostró de forma muy acusada en deseo de infidelidad sexual y conducta de infidelidad sexual, ya que la mayoría de las personas reportan fidelidad a su pareja (RiveraAragón y col., 2011; Torres y col., 2016). Fue menos acusada en búsqueda de sensaciones sexuales y actitud positiva hacia la propia infidelidad, como en otros estudios (Norbury y col., 2015; Weinstein y col., 2015; Labrecque y Whisman, 2017). Precisamente, las puntuaciones en engrandecimiento marital fueron las que más se aproximaron a la normalidad; no obstante, las puntuaciones en ninguna de las escalas siguieron una distribución normal. Al ser una distribución característica de rasgos con flexibilidad adaptativa (Fleeson y Jayawickreme, 2015), el dato puede estar indicando que el engrandecimiento marital juega un papel relevante en la adaptación de las parejas a diversos ambientes y conflictos (Gana y col., 2016).

Retomando el objetivo de contrastar un modelo para predecir conducta de infidelidad se- xual con predictores de personalidad, actitud, valoración subjetiva y deseo, se esperaba una correlación inversa entre las 2 variables de personalidad, y esta expectativa se confirmó. Una mayor tendencia hacia búsqueda de sensaciones sexuales se asocia a menor tendencia a idealizar a la pareja. Esta asociación puede deberse a que el matrimonio es vivido como una coartación de la exploración sexual y por lo tanto no se idealiza; o que la alta idealización del matrimonio inhiba la tendencia a la exploración sexual y fomente la monogamia o exclusividad sexual e incluso la rutina sexual (Matthews y col., 2018; ZeiglerHill y col., 2019).

Una de las vías direccionales del modelo proponía que la actitud positiva hacia la propia infidelidad fuera predicha por mayor búsqueda de sensaciones sexuales (Matthews y col., 2018) y menor engrandecimiento marital (Zeigler-Hill y col., 2019). Se confirmó esta expectativa con un tamaño del efecto grande al explicarse tres décimos de la varianza de esta actitud. Ambas tendencias promueven una actitud positiva hacia la propia infidelidad, con mayor peso de la primera ( $\beta$ directo $=\beta$ total $=0.444)$ que de la segunda ( $\beta$ directo $=\beta$ total $=-0.247)$. Esto podría ser explicado por un mecanismo de congruencia cognitiva o autojustificación (Jackman, 2015) o una auto-condescendencia, sin implicar que se acepte la infidelidad de la pareja (Shimberg y col., 2016).

Otra de las vías direccionales del modelo estructural era la predicción de una mayor satisfacción marital por mayor engrandecimiento marital (Gana y col., 2016; McNulty y col., 2018; Karandashev, 2019) y una actitud de mayor rechazo hacia la infidelidad marital (Silva y col., 2017). También se confirmó con un tamaño del efecto grande al explicarse más de dos quintos de la varianza de la satisfacción marital. Esta relación es fácilmente comprensible, ya que ambas variables facilitan la focalización en los aspectos positivos de la convivencia marital, incluso su amplificación y vivificación. 
La vía direccional de que la satisfacción sexual sea predicha por mayor satisfacción marital, mayor engrandecimiento marital y mayor búsqueda de sensaciones sexuales (Moral, 2009) se sostuvo, siendo el tamaño del efecto del modelo grande, con más de la mitad de su varianza explicada. La satisfacción marital con tamaño del efecto grande ( $\beta$ directo = $\beta$ total $=0.635) \mathrm{y}$ el engrandecimiento marital con tamaño del efecto total también grande $(\beta$ total $=0.584$, siendo 0.172 el efecto directo y 0.412 el indirecto) facilitan una valoración positiva de las relaciones sexuales maritales, como señalan Fugl-Meyer y col. (2019). Por otra parte, la búsqueda de sensaciones sexuales parece contribuir a enriquecer el disfrute sexual con la pareja, desde el juego sexual, la inversión de tiempo y el ensayo de novedades (Shafiee y Etemadi, 2018), aunque su efecto directo fue trivial ( $\beta$ directo $=0.087$ ) . Además, cabe señalar que su efecto indirecto fue de signo opuesto ( $\beta$ indirecto $=-0.030$ ), con lo que el efecto total finalmente no fue significativo $(\beta$ total $=0.056)$. No obstante, esta vía se requiere para un buen ajuste. Su eliminación empeora significativamente el ajuste y los índices de mejora de ajuste sugieren su inclusión, debido a la primacía del efecto de disfrute del sexo (directo) sobre el efecto atenuador de la satisfacción marital, desde una actitud poco favorable a la exclusividad marital (indirecto). Por tanto, puede que no sea un parámetro significativo en futuras investigaciones, especialmente si el tamaño de la muestra es pequeño.

El modelo propuso la predicción del deseo de infidelidad sexual a través de la satisfacción marital (Rivera-Aragón y col., 2011; Torres y col., 2016), la búsqueda de sensaciones sexuales (Kogan y col., 2015), el engrandecimiento marital (O’Rourke y Cappeliez, 2002; Starratt y col., 2017), la actitud positiva hacia la propia infidelidad (Labrecque y Whisman, 2017) y la insatisfacción sexual con el cónyuge (Scheeren y col., 2018). Los datos apoyan parcialmente esta propuesta. El efecto desinhibidor de la búsqueda de sensaciones y el facilitador de una actitud positiva hacia la propia infidelidad aumentan el deseo de sexo extradiádico, el primero con un tamaño del efecto medio $(\beta$ total $=0.489$, siendo 0.377 el efecto directo y 0.113 el indirecto), y el segundo pequeño ( $\beta$ total $=0.285$, siendo 0.274 el efecto directo y 0.011 el indirecto). Respecto al factor motivante de la insatisfacción, la satisfacción sexual tiene un efecto directo pequeño $(\beta$ directo $=\beta$ total $=-0.162)$, pero la satisfacción marital tiene un efecto directo nulo $(\beta$ directo $=-0.079, \mathrm{IC}$ al $95 \%[-0.200,0.012])$ en el modelo hipotético. La satisfacción sexual, al ser más específica y mediar el efecto indirecto de la satisfacción marital, generó que el efecto directo de la satisfacción marital no fuese significativo. Por tanto, el deseo de infidelidad sexual aumenta en la medida que la insatisfacción marital incrementa la insatisfacción sexual, con un efecto indirecto pequeño $(\beta$ indirecto $=\beta$ total $=-$ 0.103 $)$. El efecto del modelo sobre el deseo de infidelidad sexual fue grande, con más de la mitad de su varianza explicada.

Como vía direccional final del modelo se propuso que la conducta de infidelidad sexual podría ser predicha por el efecto directo del deseo de infidelidad sexual (Buss, 2016; Torres y col., 2016) y la búsqueda de sensaciones sexuales (Chen y col., 2018), y que este deseo mediaría el efecto de las demás variables. La propuesta fue apoyada por los datos, teniendo el modelo un tamaño del efecto grande sobre la conducta de infidelidad sexual, con siete décimos de su varianza explicada. El deseo actúa como un factor motivante, con un tamaño del efecto muy grande $(\beta$ directo $=\beta$ total = 0.784). El efecto total de la búsqueda de sensaciones sexuales fue medio $(\beta$ total $=0.480$ ) con mayor peso del efecto indirecto ( $\beta$ indirecto $=0.383)$ que del directo $(\beta$ directo $=0.096)$. Se ha propuesto que su efecto directo es provocado por una desinhibición de la conducta de infidelidad sexual (Chen y col., 2018). $\mathrm{Su}$ efecto indirecto en el modelo tiene dos vías. Una es el incremento del deseo de novedad sexual per se. La otra vía es más compleja, ya que parte de una actitud favorable hacia la propia infidelidad sexual, la cual disminuye 
la satisfacción marital y la satisfacción sexual con la pareja; la disminución de ambos tipos de satisfacción lleva a desear novedad sexual $y$, finalmente, a incurrir en infidelidad sexual.

En el presente modelo predictivo de conducta de infidelidad sexual la variable más crítica es el deseo de infidelidad sexual, cuyo determinante más importante es la búsqueda de sensaciones sexuales. Esta variable de rasgo se especificó como exógena, por lo que no es explicada por el modelo. No obstante, puede ser modulada y modificada, al ser un rasgo de personalidad (Dimaggio y Lysaker, 2018). Precisamente, la idealización o menosprecio a la pareja la modula en el modelo. A su vez, el modelo remarca aspectos de insatisfacción marital y sexual, los cuales pueden ser abordados en asesorías a parejas.

Se puede tomar como limitación del estudio el hecho de que las inferencias se circunscriben a la población de personas casadas residentes en Monterrey en el momento del muestreo. En relación con otras poblaciones o la misma población en otros puntos temporales, los presentes resultados constituyen datos de comparación. Otra limitación es el diseño del estudio. Esta investigación fue ex post facto transversal, sin la manipulación ni control de variables, por lo que no se pueden hacer inferencias causales. Consecuentemente, los datos sólo pueden ser interpretados en términos correlacionales y predictivos. El análisis de datos se hizo en la muestra conjunta, cuando pueden existir diferencias entre mujeres y hombres. Para futuras investigaciones se sugiere contrastar la invarianza del modelo entre los 2 sexos, pudiendo tener la búsqueda de sensaciones sexuales el papel más diferencial, ya que la cultura mexicana circunscribe la sexualidad femenina esencialmente al matrimonio y concede mucha mayor libertad a la sexualidad masculina (Hietanen y Pick, 2015).

\section{CONCLUSIONES}

El modelo psicosocial propuesto para predecir la conducta de infidelidad sexual mostró tener validez. El tamaño del efecto fue grande y el ajuste de los datos fue bueno. Es importante considerar que se aplicó a una muestra de personas casadas, residentes en la ciudad de Monterrey y entrevistadas en su domicilio. La mayoría de los entrevistados manifestaron estar satisfechos con sus matrimonios, con las relaciones sexuales con su cónyuge y tendieron a engrandecer o idealizar a la pareja. El $83 \%$ afirmó no incurrir en conductas de infidelidad sexual, $60 \%$ mostró un total rechazo hacia la infidelidad sexual, $72 \%$ no reportó deseo de infidelidad sexual y $51 \%$ no deseaba la búsqueda de sensaciones sexuales. Por otra parte, el $6 \%$ estaba insatisfecho con su matrimonio, $13 \%$ con las relaciones sexuales con su pareja y $20 \%$ veía más defectos que virtudes en sus parejas. Es conveniente aplicar el modelo en diferentes poblaciones para determinar su validez predictiva en distintos contextos.

\section{AGRADECIMIENTOS}

Se agradece al equipo que realizó el trabajo de campo y colecta de datos, así como a los participantes que colaboraron en esta investigación. El estudio fue financiado por el Programa de Apoyo a la Investigación Científica y Tecnológica de la Universidad Autónoma de Nuevo León. Clave de proyecto: CS454-14.

\section{REFERENCIAS}

APA, American Psychological Association (2017). Ethical principles of psychologists and code of conduct. With the 2016 amendment to standard 3.04. Washington, DC: APA. [En línea]. Disponible en: https://www.apa.org/ethics/code/. Fecha de consulta: 20 de octubre de 2019.

Arbuckle, J. L. (2007). AMOS 16. Analysis of moment structures. Version 16. [Software de cómputo]. Chicago, Illinois: IBM SPSS AMOS.

Bollen, K. A. and Stine, R. A. (1993). Bootstrapping goodness-of-fit measures in structural equation models. In K. A. Bollen and J. S. Long (Eds.), Testing structural equation models (pp. 111-135). Newbury Park, CA: Sage. 
Buss, D. M. (2016). The evolution of desire: Strategies of human mating (Third edition). New York: Basic books. $368 \mathrm{Pp}$.

Byrne, B. (2016). Structural equation modelling with AMOS: Basic concepts, applications, and programming (Third edition). New York: Routledge Academic. 438 Pp.

Chen, L., Yang, Y., Su, W., Zheng, L., Ding, C., and Potenza, M. N. (2018). The relationship between sexual sensation seeking and problematic Internet pornography use: A moderated mediation model examining roles of online sexual activities and the third-person effect. Journal of Behavioral Addictions. 7(3): 565-573.

Dimaggio, G. and Lysaker, P. H. (2018). A pragmatic view of disturbed self-reflection in personality disorders: implications for psychotherapy. Journal of Personality Disorders. 32(3): 311-328.

Fallis, E. E., Rehman, U. S., Woody, E. Z., and Purdon, C. (2016). The longitudinal association of relationship satisfaction and sexual satisfaction in long-term relationships. Journal of Family Psychology. 30(7): 822-831.

Fisher, H. (2016). Anatomy of love: A natural history of mating, marriage, and why we stray (Segunda edición). New York: W. W. Norton. 464 Pp.

Fleeson, W. and Jayawickreme, E. (2015). Whole trait theory. Journal of Research in Personality. 56(1): 82-92.

Fugl-Meyer, K. S., Nilsson, M. I., von Koch, L., and Ytterberg, C. (2019). Closeness and life satisfaction after six years for persons with stroke and spouses. Journal of Rehabilitation Medicine. 51(7): 492-498.

Gana, K., Saada, Y., Broc, G., Koleck, M., and Cazauvieilh, C. (2016). Dyadic cross-sectional associations between negative mood, marital idealization, and relationship quality. The Journal of Psychology. 150(7): 897-915.

Hendrick, S. S., Dicke, A., and Hendrick, C. (1998). The relationship assessment scale. Journal of Social and Personal Relationships. 15(1): 137-142.

Hietanen, A. E. and Pick, S. (2015). Gender stereotypes, sexuality, and culture in Mexico. In S. Safdar and N. Kosakowska-Berezecka (Eds.), Psychology of gender through the lens of culture (pp. 285-305). Cham, Suiza: Springer.

Hudson, W. W. (1992). The WALMYR assessment scales scoring manual. Tallahassee, FL: WALMYR Publishing Co. $62 \mathrm{Pp}$.

IBM SPSS (2016). Statistical Package for the Social Sciences. Release 24. [Software de cómputo]. Chicago, Illinois: IBM SPSS.

Jackman, M. (2015). Understanding the cheating heart: What determines infidelity intentions? Sexuality y Culture. 19(1): $72-84$.
Kalichman, S. C., Johnson, J., Adair, V. Rompa, D., Multhauf, K., and Kelly, J. (1994). Sexual Sensation-Seeking Scale: Development and predicting AIDS-risk behavior among homosexually active men. Journal of Personality Assessment. 62(3): 385-397.

Karandashev, V. (2019). Idealization and romantic beliefs in love. In V. Karandashev (Ed.), Cross-cultural perspectives on the experience and expression of love (pp. 83-98). Cham, Suiza: Springer.

Kline, R. B. (2016). Methodology in the social sciences. Principles and practice of structural equation modeling (Cuarta edición). New York, NY: Guilford Press. 534 Pp.

Kogan, S. M., Cho, J., Barnum, S. C., and Brown, G. L. (2015). Correlates of concurrent sexual partnerships among young, rural African American men. Public Health Reports. 130(4): 392-399.

Labrecque, L. T. and Whisman, M. A. (2017). Attitudes toward and prevalence of extramarital sex and descriptions of extramarital partners in the 21st century. Journal of Family Psychology. 31(7): 952-95\%.

Lammers, J. and Maner, J. (2016). Power and attraction to the counternormative aspects of infidelity. The Journal of Sex Research. 53(1): 54-63.

López-Parra, M. S., Vargas-Núñez, B. I. y Cortés-Martínez, E. (2018). Predictores de infidelidad y deseo de infidelidad sexual en relaciones premaritales. En R. Díaz-Loving, I. Reyes-Lagunes y F. López-Rosales (Eds.), La psicología social en México. Volumen XVII (pp. 611-628). Ciudad de México: Asociación Mexicana de Psicología Social.

Matthews, S. J., Giuliano, T. A., Rosa, M. N., Thomas, K. H., Swift, B. A., Ahearn, N. D., ..., and Mills, M. M. (2018). The battle against bedroom boredom: Development and validation of a brief measure of sexual novelty in relationships. The Canadian Journal of Human Sexuality. $27(3): 27 r-287$.

McIntyre, J. C., Barlow, F. K., and Hayward, L. E. (2015). Stronger sexual desires only predict bold romantic intentions and reported infidelity when self-control is low. Australian Journal of Psychology. 67(3): 178-186.

McNulty, J. K., Meltzer, A. L., Makhanova, A., and Maner, J. K. (2018). Attentional and evaluative biases help people maintain relationships by avoiding infidelity. Journal of Personality and Social Psychology. 115(1): 76-95.

Moral, J. (2009). Medida de la satisfacción sexual en parejas casadas: un estudio de validación. Archivos Hispanoamericanos de Sexología. 15(1): 46-ro.

Moral, J. (2015). Validación del modelo unidimensional de la Escala de Valoración de la Relación en personas casadas y en unión libre de Monterrey, México, en Revista 
Internacional de Psicología. [En línea]. Disponible en: http://www.revistapsicologia.org/index.php/revista/article/ view/134. Fecha de consulta: 20 de octubre de 2019.

Moral, J. (2017). Validez estructural y convergente de la Escala de Engrandecimiento Marital en México. PSICUMEX. 7(1): 35-5\%.

Moral, J. (2018a). Unidimensionalidad del Índice de Satisfacción Sexual de Hudson en adultos mexicanos casados y en unión libre. Cuadernos Hispanoamericanos de Psicología. 17(2): 28-42.

Moral, J. (2018b). Validación de la Escala de Búsqueda de Sensaciones Sexuales en mexicanos casados o cohabitantes. Revista Evaluar. 18(3): 49-72.

Moral, J. (2019). Frecuencia de relaciones sexuales y número de parejas concurrentes entre personas casadas o cohabitantes de Monterrey, Nuevo León, México. Perspectivas Sociales. 21(1): 115-145.

Norbury, A., Kurth-Nelson, Z., Winston, J. S., Roiser, J. P., and Husain, M. (2015). Dopamine regulates approach-avoidance in human sensation-seeking, in International Journal of Neuropsychopharmacology. [En línea]. Disponible en: https://doi.org/10.1093/ijnp/pyv041. Fecha de consulta: 20 de octubre de 2019.

Open Science Collaboration (2015). Estimating the reproducibility of psychological science. Science. 349(6251): 910-911.

O'Rourke, N. and Cappeliez, P. (2002). Development and validation of a couples measure of biased responding: The Marital Aggrandizement Scale. Journal of Personality Assessment. 78(1): 301-320.

Rivera-Aragón, S., Díaz-Loving, R., Villanueva-Orozco, G. B. T. y Montero-Santamaría, N. (2011). El conflicto como un predictor de la infidelidad. Acta de Investigación Psicológica. 1(2): 298-315.

Romero, A., Rivera-Aragón, S. y Díaz-Loving, R. (2007). Desarrollo del inventario multidimensional de infidelidad (IMIN). Revista Iberoamericana de Diagnóstico y Evaluación Psicológica. 23(1): 121-147.

Rosenberg, K. P. (2018). Infidelity: Why men and women cheat. New York, NY: Da Capo Lifelong Books. 288 Pp.

Scheeren, P., Ochoa-de-Alda, I., and Wagner, A. (2018). Marital infidelity: The experience of men and women. Trends in Psychology. 26(1): 355-369.

Shafiee, Z. and Etemadi, O. (2018). Predicting desire to betrayal based on romance jealous and sensation seeking in married women. Journal of Fundamentals of Mental Health. 20(2): 121-130.

Shimberg, J., Josephs, L., and Grace, L. (2016). Empathy as a mediator of attitudes toward infidelity among colle- ge students. Journal of Sex \& Marital Therapy. 42(4): 353-368.

Silva, A., Saraiva, M., Albuquerque, P. B., and Arantes, J. (2017). Relationship quality influences attitudes toward and perceptions of infidelity. Personal Relationship. 24(4): 718-728.

Stanford, P. S. (2016). Infidelity in the United States. . In C. L. Shehan (Ed.), The Wiley Blackwell encyclopedia of family studies (pp. 1320-1335). Malden, MA: John Wiley y Sons, Inc.

Starratt, V. G., Weekes-Shackelford, V., and Shackelford, T. K. (2017). Mate value both positively and negatively predicts intentions to commit an infidelity Personality and Individual Differences. 104(1): 18-22.

Torres, A. P., Torres-Castañeda, L. J. y Riveros-Munevar, F. (2016). Relación entre tendencia a la infidelidad emocional y/o sexual e inteligencia emocional, en estudiantes universitarios. Cuadernos Hispanoamericanos de Psicología. 16(1): 57-70.

Weinstein, A., Katz, L., Eberhardt, H., Cohen, K., and Lejoyeux, M. (2015). Sexual compulsion - Relationship with sex, attachment and sexual orientation. Journal of Behavioral Addictions. 4(1): 22-26.

Weis, D. L. and Fenton, J. R. (1987). Marital exclusivity and the potential for future marital conflict. Social Work. 32(1): 45-49.

Yucel, D. and Koydemir, S. (2015) Predictors of marital satisfaction in North Cyprus: exploring the gender effects. Journal of Family Studies. 21(2): 120-143.

Zapien, N. M. (2017). Decision science, risk perception, and infidelity. SAGE Open. [En línea]. Disponible en: https://doi.org/10.117r/2158244016686810. Fecha de consulta: 20 de octubre de 2019.

Zeigler-Hill, V. Seidman, G., Trombly, D. R., Ishaq, J., Shango, R., and Roberts, A. (2019). Narcissism and romantic commitment: The role of attitudes toward alternative romantic partners, in Self and Identity. [En línea]. Disponible en: https://doi.org/10.1080/15298868.2019.1635521. Fecha de consulta: 20 de octubre de 2019. 